\title{
Measurements of interannual variation of the vertical at Jozefoslaw by astrometric and gravimetric observations
}

\section{Z.X. Li}

Shanghai Observatory, Chinese Academy of Sciences Shanghai 200030, China

e-mail: lzx@center.shao.ac.cn

\begin{abstract}
Jozefoslaw astronomical and geodetical observatory at Warsaw is the place where parallel observations of astrometric latitude (since 1959) and meridional plumb line variations from gravimetric methods (since 1976) have been carried out continuously over the past 20 years. The observational data at this observatory have been analysed to confirm the reality of the plumb line variation results derived from the astrometric latitude residuals. Cross correlation analyses between the results of the two techniques have demonstrated that the interannual plumb line variations along the meridian detected by the astrometric technique is in good accordance with those from the gravimetric technique. The results shown in the paper can be considered as evidence of the existence of non-tidal plumb line variations at interannual time scales, of which the scale is about $0.02^{\prime \prime}$ in the case of Jozefoslaw observatory, and the possibility in measuring them by astrometric techniques.
\end{abstract}

Key words: earth — reference systems — astrometry

\section{Introduction}

Optical observations of astronomical time and latitude are based on the local plumb line (vertical) at the observational site. Variations of the plumb line are considered as errors in astronomical observations. On the contrary, plumb line variation (PLV) related to the deformation of the geoid is an important scientific object capable of being measured in practice, with which geodynamics and some other sciences may be studied in a better way. Thus, both the astronomical and geodetical communities have long been interesting in measuring the PLV. Due to the difficulties in separating it from the other sources, such as star catalog error, observational error caused by atmospheric refraction, and the inaccuracy of the ERP determination, the problem remains unsolved. The high accuracy of ERP determination by space techniques since 1976,

Send offprint requests to: $\mathrm{Z} . \mathrm{X}$. $\mathrm{Li}$ the promising prospect of a high-precision catalog after the Hipparcos Space Mission, the possibility of estimating atmospheric refraction by the NASA global atmospheric data (Schubert et al. 1993), all of these progresses in science and technology will soon provide us a new foundation in studying PLV.

New efforts have already been put into practice by the IAU and IAG since the beginning of the 1990's. As a first step, studies concentrate on the possibility of deriving long-term variations in the deflection of the vertical from astrometric observations (IAU Comm. 19 1991). Other than the efforts already made recently (Ishii et al. 1990; Li \& Gambis 1994; Li 1995, 1996), one of the best ways to confirm the actual ability of astrometric techniques in measuring the PLV is to compare their results directly to the corresponding results derived from another technique. Fortunately, the Astronomical-Geodetic Observatory at Jozefoslaw is the place where regular latitude observations have been carried out since 1961, and gravimetric measurements have been used to calculate the PLV along the meridian there since 1976 (Rogowski \& Barlik 1993). Thus, there are already 20 years of parallel observations of the two techniques concerned with the determination of PLV. The purpose of this paper is to compare the PLV results of the two techniques, from which a conclusion might be drawn on the reality of PLV results derived from the two techniques.

We restrict ourself here only to the interannual part of the PLV, mainly due to two points: the existence of star catalog errors in the astrometric observations, which are an order greater than the PLV we are going to detect; the sparse distribution of the gravimetric measurements in the case of Jozefoslaw observatory.

With the restriction of the data used in the paper, it is difficult for us to solve all of the problems in one paper. We expect that the evidence shown in the paper and an understanding of the errors of astrometric observation of the PLV at the site of an observatory, their existence in latitude or time observations, their possible influnce on astronomical studies already done in the past, and the 
ability of astrometric techniques in measuring them at interannual time scales, may be recognized.

\section{Data and data processing}

Latitude observations at Jozefoslaw are made since 1959 with a visual zenith telescope (Zeiss No. 17224), using the Horrebow-Talcott method. A 12-group program, which has been revised twice in the past years, is used in routine observations. On average, about 150 group results are obtained per year, and the precision of a group result is on the order of $0.20^{\prime \prime}$. To monitor the stability of the plumb line, a meridian gravimetric base-line of 7 points was established in 1976, with which the variations of the meridian component of the plumb line are measured 3-4 times a year basing on the measurements of differences in gravity accelerations. Scintrex, Sodin and Worden-Master precise quartz gravimeters had been used in the early stages of the gravimetric observations, with precision of the PLV on the order of $0.002^{\prime \prime}$. Lacoste-Romberg's gravimeter Model G No. 432 has been used since September 1987 and the precision has been improved (Barlik \& Rogowski 1989).

The data used in this study are shown in Table 1, in which $N$ is the number of the observations.

Table 1. Data used in the study

\begin{tabular}{llll}
\hline Code & Type & Time Interval & $N$ \\
\hline LO & Latitude observations & $1976-1995$ & 3028 \\
PL & Plumb line variations & $1976-1995$ & 77 \\
\hline
\end{tabular}

The reduction method for obtaining a latitude residual (LR) is the same as the one used in the previous work ( $\mathrm{Li}$ 1996), but the polar motion results used in obtaining an LR time series are those from space techniques (Gross 1995).

To compare the LR and the PL with the same dates, a weighted mean of LR is first calculated at 30-day intervals. The new LR time series is $\operatorname{smoothed}(e=-11)$ (Vondrak 1969, 1977) and then interpolated at the same 77 dates of the PL time series. The 77-point time series of the LR(77) is then compared with the PL time series (see Fig. 1).

The band filters used and the calculating of the crosscorrelation function between the $\mathrm{LR}(77)$ and the PL time series are the same as those used in the previous paper $(\mathrm{Li}$ 1996).

\section{Results}

After using three different bands in filtering, crosscorrelation analyses of the two filtered time series have been made. Figure 2 shows the three different results in
Observational Data and Data Processing

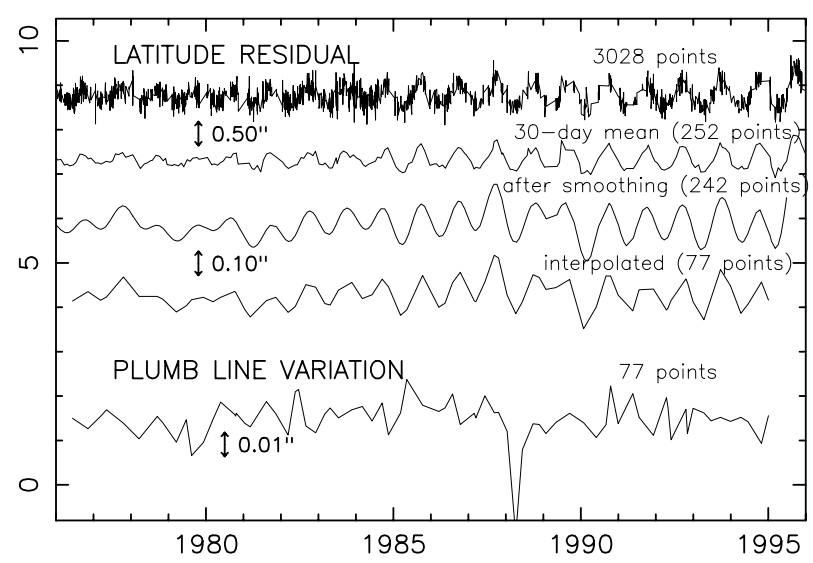

Fig. 1. The PL time series and the processing of the LR time series

using solid, dash, and dot lines, respectively. The two cutting points in days of a band filter are expressed nearby the corresponding straight line. The level of significance, expressed by the straight line, comes from a Monte Carlo simulation done under the same conditions as that of the actual two time series.

\section{With Different Band Filters}

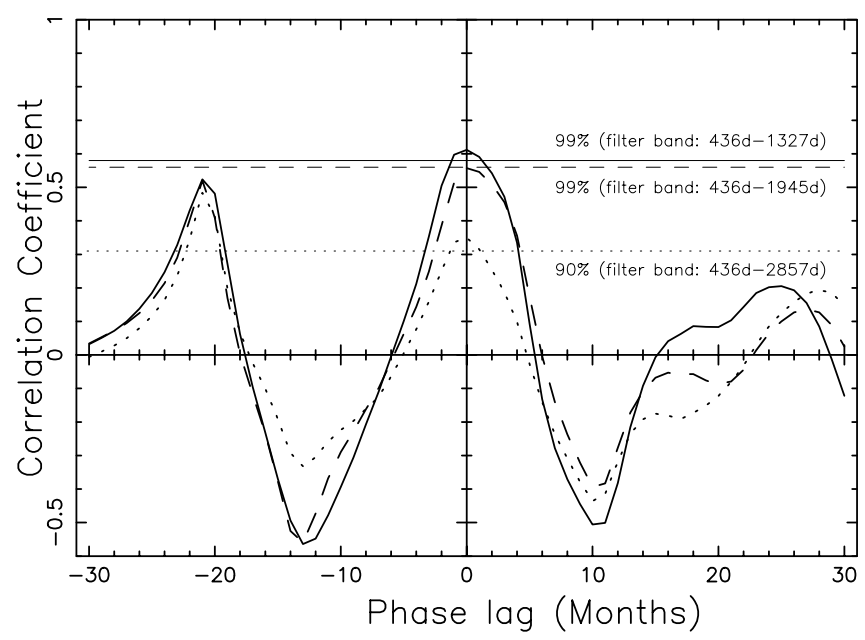

Fig. 2. Cross correlation between $\operatorname{LR}(77)$ and PL time series as a function of phase lag at interannual time scales

Direct comparisons of the filtered time series in using three different band filters are shown in Fig. 3.

\section{Discussion}

1. From Fig. 2, the correlation at interannual time scales is quite evident between the results of the two different 
With Different Band Filters

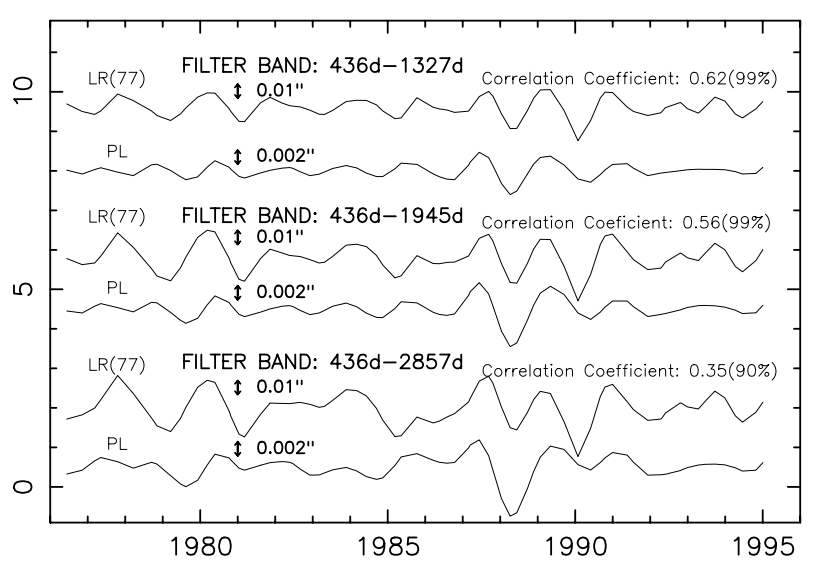

Fig. 3. Comparison between $\operatorname{LR}(77)$ and PL time series in no phase lag at interannual time scales

techniques. The similarities of astrometric and gravimetric curves shown in Fig. 3 further enhance the reality of the correlationship. There is a scale difference between the two derived results, which will be explained below.

2. In gravimetry, the Vening-Meinesz formula is used to estimate the plumb line deflection $\Delta \xi$ at an observatory (Barlik \& Rogowski 1989):

$\Delta \xi=-\frac{1}{2 \pi} \int_{0}^{2 \pi} \int_{0}^{\pi} \Delta g Q(\psi) \cos A \mathrm{~d} \psi \mathrm{d} A$

in which, $A$ denotes the azimuth from the observatory to the point with a given gravity anomaly $\Delta g$, $\psi$ the angular distance, and $Q(\psi)$ the Vening-Meinesz function. In the case at Jozefoslaw observatory, the anomaly integration range has been limited to a very small angular distance $R(R=15 \mathrm{~km}=0.00235$ radian). The estimate $\Delta \xi_{1}$ obtained in such a case, different from the real one $\Delta \xi$, and its corresponding correction $\Delta \xi_{2}$ are the following:

$\Delta \xi_{1}=-\frac{1}{2 \pi} \int_{0}^{2 \pi} \int_{0}^{R} \Delta g Q(\psi) \cos A \mathrm{~d} \psi \mathrm{d} A$
$\Delta \xi_{2}=-\frac{1}{2 \pi} \int_{0}^{2 \pi} \int_{R}^{\pi} \Delta g Q(\psi) \cos A \mathrm{~d} \psi \mathrm{d} A$.

From a simulation under the assumption that the anomalies, within a $75 \mathrm{~km}$ range, are correlated as $\Delta g_{i}=\Delta g_{0}+K \psi_{i} \cos A_{i}$, where $\Delta g_{0}$ denotes the anomaly at the observatory and $K$ is a constant, the scale of the correction $\Delta \xi_{2}$ will be four times greater than that of $\Delta \xi_{1}$ obtained. Thus, the scale difference shown in Fig. 3 is possible in practice.

3 . The only common component existing in both the latitude residuals and the PLV from the gravimetric technique is the PLV itself at Jozefoslaw observatory. From the correlationship, including the level of significance and zero phase lag between the two time series derived from different techniques, one can conclude that the result of the interannual PLV measured by the astrometric technique is reliable.

\section{Conclusions}

From the comparison between the PLV results derived from the astrometric and gavimetric techniques at interannual time scales, one can conclude that the interannaul PLV along the meridian is in the order of $0.02^{\prime \prime}$ in the case of Jozefoslaw observatory, and it is possible to measure it in practice by the astrometric technique.

Acknowledgements. The author is indebted to Prof. Marcin Barlik of the Warsaw University of Technology for his kind provision of the plumb line variation results at Jozefoslaw Observatory and the related informations. Without his help, the work could not have been done. The author is also grateful to Dr. F. Barlier for his helpful comments. The study has been supported by the Chinese National Natural Sciences Foundation No. 19373018 and 19673011, and also the NSF International Program Grant through the University of Texas, Department of Geological Sciences, Professor Clark R. Wilson (Principal Investigator).

\section{References}

Barlik M., Rogowski J.B., 1989, Prace naukowe politechniki warszawskiej Z. 33, 19

Gross R.S., 1995, IERS Technical Note 21, pp. C1-C4

IAU Comm. 19, 1991, Application of optical astrometry time and latitude programs

Ishii H., Goto Y., Ishikawa T., 1990, A\&AS 86, 252

Li Z.X., Gambis D., 1994, A\&A 290, 1001

Li Z.X., 1995, Acta Astron. Sin. 36, 47

Li Z.X., 1996, A\&A 309, 313

Rogowski J.B., Barlik M., 1993, Wiss. Z. Techn. Univers. Dresden 42 H.6, 57

Schubert S.D., Pfaendtner J., Rood R., 1993, Bull. Am Met. Soc. 74,2331

Vondrak J., 1969, Bull. Astron. Inst. Cze. 20, 349

Vondrak J., 1977, Bull. Astron. Inst. Cze. 28, 84 